ARTICLE

\title{
Improvement of Neutronics Calculation Methods for Fast Reactors
}

\author{
Toshikazu TAKEDA* \\ University of Fukui, Research Institute of Nuclear Engineering, \\ 3-9-1 Bunkyo, Fukui-shi, Fukui, 910-8507, Japan
}

To accurately estimate neutronics properties of fast reactors, particularly Japan Sodium-cooled Fast Reactor of 1,500 MW electric, calculational methods are being improved in Japan.

This paper describes the planning and the ongoing development of the neutronics calculation methods in the field of 1) assembly calculations including the calculations of effective cross sections, 2) core calculations and 3) uncertainty evaluation and uncertainty reduction.

KEYWORDS: neutronics calculation, fast reactor, annular fuel, effective cross section, transport calculation

\section{Introduction}

In Japan, Monju restarted on May 2010 and the start-up test is being carried out for the three cores with $0 \%, 40 \%$, and $100 \%$ power. The fuels contain a few percent Am-241 produced by the $\beta$ decay of $\mathrm{Pu}-241$, and the measured neutronics data are very useful to valididate the cross sections of minor actinides (MAs).

The commercial type Japan Sodium-cooled Fast Reactor (JSFR) is planned to operate in 2050. The power is 1,500 MW electric, and the core contains fuel assemblies with inner duct and large annular fuel.

To accurately estimate neutronics properties of fast reactors, particularly JSFR, calculational methods have to be improved.

This paper describes the planning and the ongoing development of the neutronics calculation methods in the field of 1) cell and assembly calculations including effective cross section calculations, 2) core calculations and 3) uncertainty evaluation and uncertainty reduction.

\section{Cell and Assembly Calculations}

In cell or assembly calculations, 70 group effective cross sections have been usually calculated using the Bondarenko type self-shielding factor. The heterogeneous effect is taken into account using the equivalence theory. The 70 group cross sections or the collapsed cross sections are used for core calculations. Diffusion theory is usually utilized to calculate neutronic characteristics, and transport theory is used to obtain the transport correction to the results of the diffusion theory calculations. This calculation procedure has been commonly used for fast reactor analysis in Japan.

However, in the procedure, the detailed neutron spectrum is not taken into account in calculating effective cross sections. Furthermore, the interference effect to the neutron

*Corresponding author,E-mail:t_takeda@u-fukui.ac.jp

(C) 2011 Atomic Energy Society of Japan, All Rights Reserved. spectrum between resonances of different isotopes is not considered. To overcome these problems, the SLAROM-UF ${ }^{1)}$ has been developed where the ultra-fine group is adopted in cell calculations.

The nuclear data library JENDL-3.3 ${ }^{2)}$ has been commonly utilized.

However, JENDL-4.0 $0^{3)}$ was released. So, the nuclear data library JENDL-4.0 will be used instead of JENDL-3.3 in the near future because the core performances of fast reactors calculated by JENDL-4.0 agree well with the measured data.

In SLAROM-UF, 250 70 group effective cross sections are calculated using the ring model of a fuel assembly. However, it would be difficult to represent the fuel assembly with inner duct structure (FAISUS), which is currently considered for JSFR, in a one-dimensional cylindrical model because of its non-uniform structure. So, we are planning to compare the effective cross sections calculated by using several one-dimensional assembly models like that including a marginal cyrindrical duct and central cyrindrical duct. To calculate the effective cross sections of annular fuel pellets, we are considering the following method.

\section{Dancoff Factor Method ${ }^{4)}$}

The Dancoff factor for a black annular fuel is the same as that for a black solid fuel. In the method, the background cross section is calculated by

$$
\sigma^{n}=\sigma^{n^{*}}+\frac{a \Sigma_{e}}{N_{n}} \frac{1-C}{1-C+a C}
$$

where $\sigma^{n^{*}}$ is the background cross section for fuel region, and

$$
\begin{aligned}
& a: \text { Bell factor } \\
& C: \text { Dancoff factor } \\
& \Sigma_{e}=1 / l \\
& l: \text { mean chord length for fuel rod }
\end{aligned}
$$

The mean chord length corresponds to the diameter $\left(2 r_{1}\right.$ 


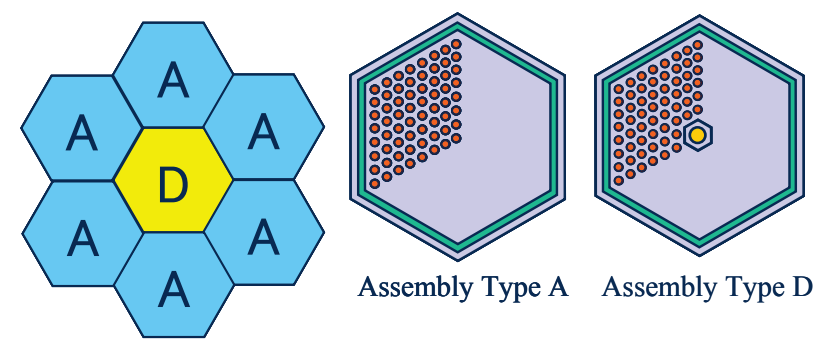

Fig. 1 A color set model

where $r_{1}$ is fuel radius) for a solid fuel rod, and to $2\left(r_{1}-r_{0}\right)$ for an annular fuel rod where $r_{0}$ is the inner hole radius.

\section{Stocker-Weiss Method ${ }^{5)}$}

The Stocker-Weiss method can treat the spatial dependence of the self-shielding factor of cross sections.

\section{Subgroup Method ${ }^{6-7)}$}

In this method, each energy group is subdivided into a few subgroups. As subgroup parameters, the representative cross section and the probability for each sub-group are evaluated. Subgroup fluxes are calculated using the subgroup parameters, and are used to calculate the group averaged cross sections. The sub group method has been applied to a representative Monju fuel, and it was found that the calculated cross sections are almost the same as calculated from the Dancoff factor method.

We are now planning to apply the method to the fuels of JSFR, and to investigate the effect of the spatial distribution of the self-shielding effect.

The effective cross sections thus calculated are used in assembly calculations to obtain assembly homogenized cross sections. Usually, infinite assembly calculations will be carried out for fuel assemblies. However, for special assemblies such as control rod assemblies, control rod position assemblies, blanket assemblies, color set models are used which contain the special assembly and adjacent fuel assemblies as shown in Fig. 1. To treat the heterogeneity in assemblies, the $\mathrm{BACH}$ code $^{8)}$ based on the method of characteristics on hexagonal geometry will be used for the calculations.

In order to accurately calculate assembly-averaged reaction rates, the SPH (spatial homogenization) factor is calculated. ${ }^{8)}$ First, a heterogeneous MOC calculation is carried out for the color set. After the heterogeneous MOC calculation, homogeneous MOC calculations are performed with assembly-averaged cross sections:

$$
\begin{gathered}
\bar{\Sigma}_{i}^{(n)}=\frac{\int_{v i} d \vec{r} \Sigma(r) \phi(r)}{\int_{v i} d \vec{r} \phi^{(n-1)}(r)} \\
=f_{i} \overline{\Sigma_{i}}
\end{gathered}
$$

where $\phi(r)$ is the flux obtained by the heterogeneous MOC calculation, and $\phi^{(n-1)}(r)$ is the flux calculated by using ${\overline{\Sigma_{i}}}^{(n-1)}, \bar{\Sigma}_{i}$ is the conventional flux weighted assembly av-
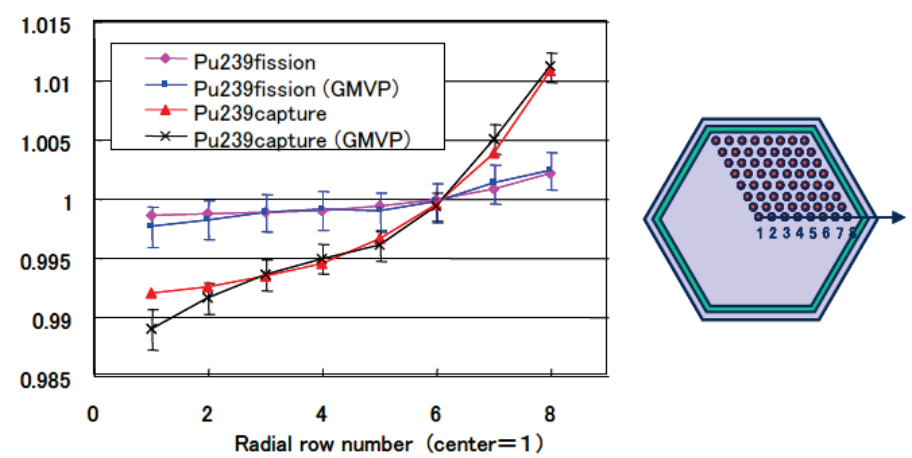

Fig. 2 Normalized reaction rate distribution for infinite assembly calculation

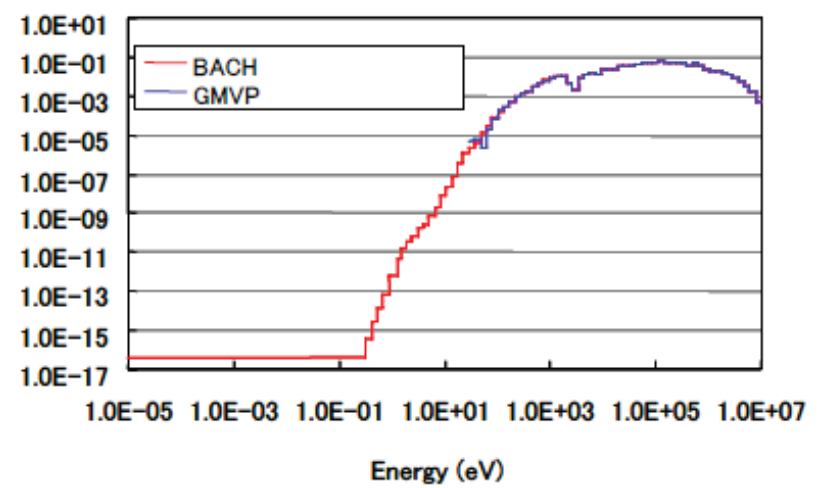

Fig. 3 Neutron spectrum at the center of the assembly D for the color set model shown in Fig. 1

eraged cross section, and $f_{i}$ is the $\mathrm{SPH}$ factor.

This MOC calculation is carried out until a convergence is achieved for the assembly-averaged fluxes $\phi^{(n)}$.

Let us show some example of heterogeneous MOC calculations. Figure 2 shows the comparison of reaction rate distributions for $\mathrm{Pu}-239$ fission and capture within a fuel assembly calculated by the MOC code BACH and the Monte Carlo code GMVP. ${ }^{9)}$ The calculations were performed in 70 groups. A good agreement in seen, and the standard deviation of GMVP is large at the innermost fuel pin because neutron tally is small for the pin. BACH yields a reasonable smooth distribution.

Next we have applied the BACH code to color set shown in Fig. 1. Figure 3 shows the neutron spectrum at the center fuel of the assembly D.

The neutron spectrum calculated by $\mathrm{BACH}$ is in good agreement with that calculated by GMVP above $10 \mathrm{eV}$. It is difficult to obtain the neutron spectrum below $10 \mathrm{eV}$ by the Monte-Carlo method because many of neutrons are captured by U-238, and the neutron tally number becomes small for the energy range. The deterministic codes (BACH code) do not suffer this difficulty.

Finally BACH has been applied to a 2-dimensional core calculation considering the pin-by-pin heterogeneity of each assembly. Figure 4 shows the calculational geometry. Calculations were performed in 7 groups. The $k_{\text {eff }}$ results are shown in Table 1. In the Monte-Carlo calculation, the histo- 


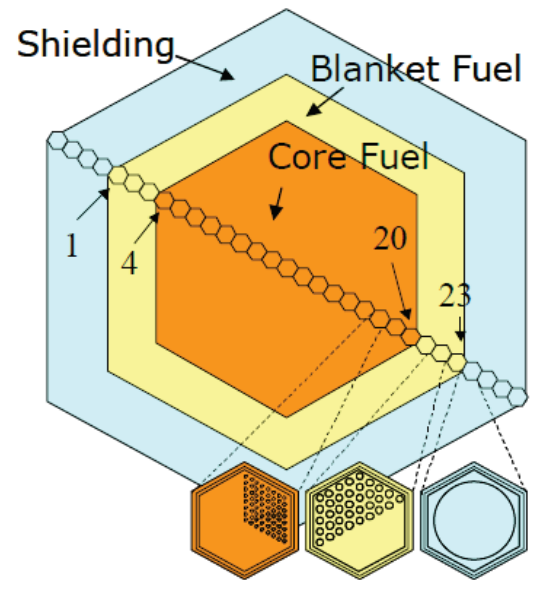

Fig. 4 FBR core model

Table 1 Comparison of $k_{\text {eff }}$ in FBR core

\begin{tabular}{|c|c|}
\hline Method & keff \\
\hline $\begin{array}{c}\text { GMVP* } \\
\text { (Monte-Carlo) }\end{array}$ & $1.15160 \pm 0.004 \% \Delta \mathrm{k}$ \\
BACH & 1.15270 (Difference of $0.10 \% \Delta \mathrm{k})$ \\
\hline
\end{tabular}

* neutron history : 100 millions

ries number was set to 100 millions to reduce the standard deviation. BACH result agrees with GMVP with the small difference of $0.10 \% \Delta k$, and this agreement verifies the accuracy of the BACH code.

\section{Core Calculations}

In core calculations, we are planning to use the homogenized cross sections calculated by BACH. The SPH factors calculated from the color set models are also used in the calculations. Thus the heterogeneous effect is included in the homogeneous core calculations.

The homogeneous core calculations will be performed by using the NSHEX code ${ }^{10}$ which is based on the Sn nodal method. The accuracy of NSHEX was verified for the small fast reactors and the Monju. For JSFR we have to check the accuracy of the method: The effect of the angular quadrature and nodal expansion will be investigated. The core performance parameters calculated by NSHEX will be compared with those by the Monte-Carlo method for JSFR.

In the core calculations performed by the nodal transport code NSHEX. The pin-by-pin power distribution is not calculated. To calculate the pin-by-pin power distribution, the 3-D transport calculation method is being developed based on the method of characteristics (MOC). In the results of 2-D pin-by-pin calculations, there were some unreasonable flux distributions in the Monte-Carlo method. However, there were no unreasonable results seen for the MOC flux

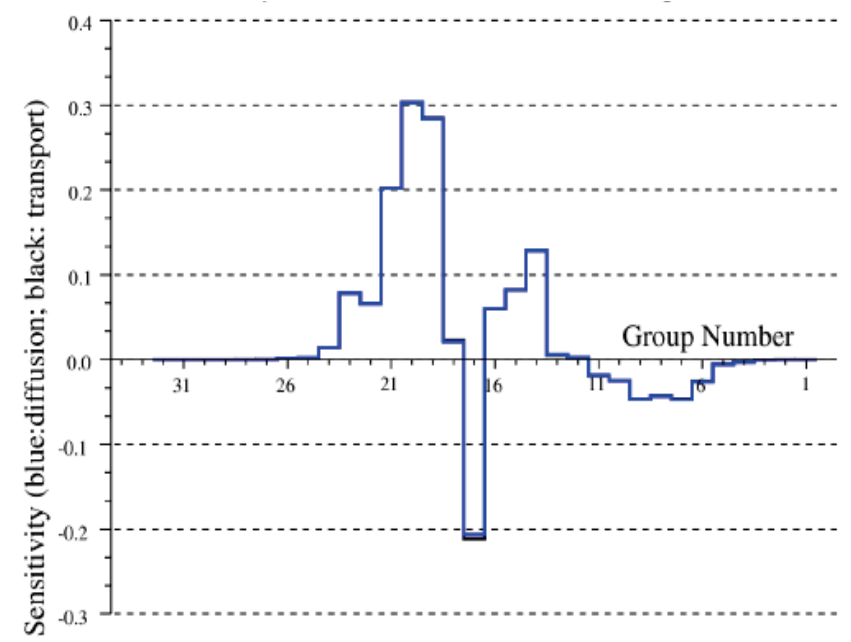

(a) U-238 capture cross section change for JSFR

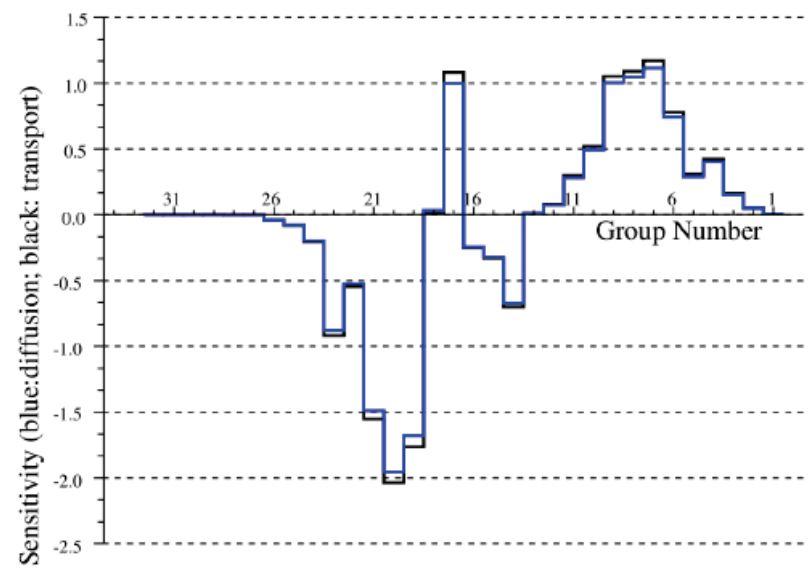

(b) Pu-239cross section change for Monju-like core

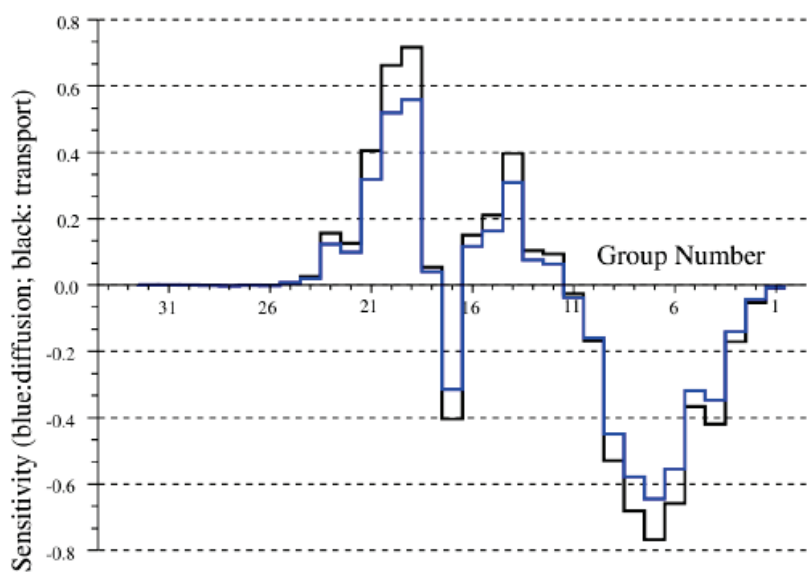

(c) $\mathrm{Pu}-239$ cross section change for small core

Fig. 5 Comparison of sensitivity coefficient for $\mathrm{Na}$ void worth

distribution. So, one can conclude that the MOC method is more reliable than the Monte-Carlo method in pin by pin power calculations. 


\section{Uncertainty Analysis and Uncertainty Reduction}

The uncertainty of the neutronics characteristics calculated by the above method and data should be within the target uncertainty. The target uncertainty of $k_{\text {eff }}$, for example, is about $0.3 \% \Delta k / k$ for Monju and JSFR. To calculate the uncertainly of the neutronics characteristics, it is necessary to perform the sensitivity and uncertainly analyses.

In performing the sensitivity and uncertainly analyses, sensitivity coefficients are required. So, we have investigated whether the transport theory calculation is necessary in calculating the sensitivity coefficients. Figure 5 compares the sensitivity coefficients of $\mathrm{Na}$ void reactivity calculated by the transport and diffusion theories. The results by the diffusion and transport calculations are shown by the blue and black lines, respectively.

From the comparison it is seen that the difference between the sensitivity coefficients calculated by the two theories is rather small for MONJU and JSFR. So, we decided to use the sensitivity calculation code SAGEP based on the diffusion theory for JSFR analysis.

The uncertainly of the calculated neutronics characteristics $R$ can be evaluated as the sum of contribution of the modeling error and the cross section error. The variance of $R$ is given by

$$
V(R)=G M G^{+}+V m
$$

where $G$ : sensitivity coefficient of $R$ with respect to cross section change

$M:$ cross section covariance

$\mathrm{Vm}$ : variance for modeling error

To reduce the uncertainly there are two procedures: the cross section adjustment method and the bias factor method.

In the cross section adjustment, various integral data for various reactors and critical assemblies can be used to adjust the cross section data. The adjusted data can be utilized in revising a cross section set, and also can be effectively utilized to the design calculation of a new core such as JSFR. The advantage of the method is the possibility of cross section adjustment over the wide range of neutron energy by using measured data for various neutron spectrum.

While, the bias factor method uses measured data for a few (or only one) mockup cores. When the mockup core is very similar to a target core which one wants to design, a good accuracy can be obtained in designing the target core. However, if there are some differences in fuels, materials, and in core sizes, there remains some uncertainties in the bias factor method. Because JSFR core is large and the core may contain MA loaded MOX fuel, it is difficult to carry out a critical experiment using a core similar to JSFR. Therefore we are considering to utilize the cross section adjustment method to reduce the design uncertainty of JSFR.

Usually the uncertainty of neutronic characteristics caused by the cross section uncertainty is larger than that by the modeling uncertainty. The cross section set $T_{0}$ can be adjusted by the use of measured data $\operatorname{Re}$ as follows:

$$
T=T_{0}+M G^{+}\left[G M G^{+}+V e+V m\right]^{-1}\left[\operatorname{Re}-R c\left(T_{0}\right)\right]
$$

where $G:$ Sensitivity coefficient

$M:$ Cross section covariance before adjustment

$R c$ : Calculated value of neutronics characteristics

$V e$ : Experimental error

$\mathrm{Vm}$ : Modeling error

The adjusted cross section $T$ has the covariance $M^{\prime}$ given by

$$
M^{\prime}=M-M G^{+}\left[G M G^{+}+V e+V m\right]^{-1} G M
$$

If the modeling error and the experimental error are zero, $M^{\prime}$ approaches zero especially when $G$ approaches square matrix. So, in this case, we can improve the cross sections remarkably.

The adjusted cross section can be utilized to designing a new target core such as JSFR and $4 \mathrm{~S}$ reactor. The design nominal values of neutronic characteristics are obtained by

$$
R c^{(2)}(T)=R c^{(2)}\left(T_{0}\right)+G^{(2)}\left(T-T_{0}\right)
$$

where superfix 2 shows the target core. The variance of the predicted value by Eq. (6) is given by

$$
V\left[R c^{(2)}(T)\right]=G^{(2)} M^{\prime} G^{(2)}+V m^{(2)}-N V m^{(12)}-V m^{(12)+} N^{+}
$$

where the superfix (12) shows the correlation between the integral experiments used for the adjustment and the design target core, and $N$ is given by

$$
N=G^{(2)} M G^{(1)}\left[G^{(1)} M G^{(1)+}+V e^{(1)}+V m^{(1)}\right]^{-1}
$$

From Eq. (7) it is seen that the prediction error of the target core is reduced when there is a strong correlation of the modeling errors between the integral experiments and the target core. We are planning to recommend to use Eq. (7) for the design of JSFR.

Furthermore, to achieve target accuracy with the existing experimental data, we are considering to use JENDL-4.0 for cross section adjustment.

\section{Conclusion}

We have described the planning and ongoing development of the neutronics calculation method covering the field of cell and assembly calculations, core calculations and uncertainty evaluation and uncertainty reduction.

The new nuclear data and the new calculation method is used for the proposed calculation system.

\section{References}

1) T. Hazama et al., SLAROM-UF: Ultra-Fine Group Cell Calculator Code for Fast Reactors, JNC TN9520 2004-01 (2004), [in Japanese].

2) K. Shibata et al., "Japanese Evaluated Nuclear Data Library Version 3 Revision-3: JENDL-3.3," J. Nucl. Soc. Technol., 39[11], 1125-1136 (2002).

3) K. Shibata et al., "JENDL-4.0: A New Library for Nuclear Science and Engineering," J. Nucl. Soc. Technol., 48[1], 1-31 (2011).

4) S. Ono et al., "CASUP: Cell Calculation Code for Fast Reactor Analysis," Tech. Reports of the Osaka University., 33[1708], 207-219 (1983). 
5) C. C. Stoker, Z. J. Weiss, "Spatially dependent resonance cross-section in a fuel rod," Ann. Nucl. Energy, 23[9], 765-778 (1996).

6) Y. Ronen, Handbook of Nuclear Reactors Calculations, CRC Press, United States (1986).

7) T. Takeda et al., "Application of Multiband Method to KUCA Tight-Pitch Lattice Analysis," J. Nucl. Sci. Technol., 28[9], 863-869 (1991).

8) T. Takeda et al., "Development of 3-D Detailed FBR Core Calculation Method Based on Method of Characteristics," Proc. Mathematics \& Computation, Supercomputing, Reactor
Physics and Biological Applications, Sep.12-15, 2005, Avignon, France (2005), [CD-ROM].

9) T. Mori et al., MVP/GMVP : General Purpose Monte Carlo Codes for Neutron and Photon Transport Calculations based on Continuous Energy and Multi Group Methods, JAERI-Data/Code 94-007, Japan Atomic Energy Research Institute (JAERI) (1994).

10) H. Ikeda, T. Takeda, "A New Nodal $S_{N}$ Transport Method for Three-Dimensional hexagonal Geometry," J. Nucl. Soc. Technol., 31[6], 467-509 (1994). 